\title{
ABET-DRIVEN GRADING AND STATISTICAL ONLINE TOOLS FOR COURSE OUTCOMES ASSESSMENT
}

\author{
Haydar Moussalem ${ }^{1}$, Amin Haj-Ali ${ }^{1}$, Hiba Alawieh ${ }^{1}$, Mohamad Merhi ${ }^{2}$, Mohamad Hajj-Hassan ${ }^{3}$, \\ Houssein Hajj-Hassan ${ }^{3}$ and Bassam Hussein ${ }^{3 *}$ \\ ${ }^{1}$ Lebanese International University, Beirut, Lebanon \\ ${ }^{2}$ Brunel University, Middlesex, United Kingdom \\ ${ }^{3}$ The International University of Beirut, Beirut, Lebanon
}

\begin{abstract}
Working towards ABET accreditation; it is indispensable to conduct course assessment to improve student learning, performance, and the educational process. Assessment determines whether or not the goals of teaching and learning are being met and triggers needed corrective measures. It provides indicators to instructors and course coordinators to assess the attainment of course outcomes (COs) and consequently the overall program outcomes in order to adapt the teaching methodologies and techniques to address any anomalies. In this paper, we report the integration of the course assessment process into the university management system (UMS). Two tools are presented, the course grading tool for individual class sections and the coordinator statistical tool for all offered class sections. The course class section grading tool is used by the instructor to assess the students' performance on the designated course activities and generate an assessment report. The coordinator statistical tool is used to collect all offered course sections data and provides a report including the course activities statistics. The coordinator uses this report to analyze the results, synthesize the instructor recommendations, and propose corrective actions to close the loop of continuous improvement. Both tools are interrelated and represent an opportunity for instructors and course coordinators to assess their students and consequently make significant and positive changes to teaching and learning based on best practices in higher education. As a case study, data from 31 sections of a basic Electric Circuits course were collected for a detailed statistical analysis.
\end{abstract}

\section{KEYWORDS}

Course assessment, Course Outcomes, ABET, Student Learning, Continuous Improvement, Best Educational Practices

\section{INTRODUCTION}

Student assessment is a critical aspect of the teaching and learning process especially at the higher education level. It usually involves processes that identify, collect, and prepare data to evaluate the attainment of student outcomes and program educational objectives. The assessment process should be tightly coupled with interpretation of the data and evidence accumulated through the assessment processes (Tarhini, Jackson, and Zelmanowitz, 2012). Assessment can be formative or summative and data gathered from both types of assessment enable instructors and course coordinators to monitor progress towards achieving the desired learning objectives (O'Neill, Moore, and McMullin, 2005; Kennedy, Hyland, and Ryan, 2006).

Presently, it is evident that in order to sustain, evolve, and improve an assessment process, automated assessment tools are needed (Hazen, Wu, and Sankar, 2012; Hajj-Hassan, Hussein, Chahine, and Haj-Ali, 2014). Automated tools are also helpful enablers in adhering to ABET guidelines when seeking accreditation (Clark, Froyd, Merton, and Richardson, 2004; Oakley, Felder, Brent, and Elhajj, 2004). ABET is recognized as a worldwide leader in assuring quality and stimulating innovation in applied science, computing, engineering, and engineering technology education (Hussein, Abdul-Nabi, Merhi, and Haj-Ali, 2015). It accredits college and university programs in the disciplines of applied science, computing, engineering, and engineering technology at the associate, bachelor, and master degree levels (Accreditation Board for Engineering and Technology (ABET)). Here, we report the development of an automated course grading software tool which primarily involves the generation of activities grades for each conducted course

*Corresponding author: bassam.hussein@b-iu.edu.lb 
evaluation activity and a statistical tool that collects all class sections data and provides a statistical report including, among others, the overall average, median, mean, and percentile grades. Both tools have been developed using the best practices in educational theories and are fully aligned with ABET guidelines for engineering programs accreditation.

\section{SYSTEM OVERVIEW}

The tools are integrated in an automated ecosystem which employs a modular architecture as depicted in Figure 1. All system components are supported by reliable, secure, and scalable infrastructure. The core meta-data stores contain the information about the programs, courses, and all pedagogical relationships. Tools and utilities are implemented to support the management of this meta-data with referential integrity at its core quality measure. In addition, the system contains a module for assessment activity and survey management. This module represents the dynamic data management subsystem. It holds data collected from assessment activities, surveys, and other tools used in data collection. At the epicenter of this tool, lies the engine responsible for mapping the dynamic data based on the static mapping of the programs and their constituents.

Data collection and processing would only serve purpose when presented in actionable reports. Therefore, the system implements a robust yet configurable reporting tool. This tool produces the basic reports required for assessment and decision-making. The reports are divided into static and dynamic formats. Static reports include syllabi, program outcomes, course program mapping etc. Dynamic reports are based on the periodic data collected including assessment activities and surveys. Dynamic reports include grading reports (from the element level up to the course level), outcome fulfillment, student success (rubric based), employability, etc... In addition, the reporting tool has all the basic to produce the quantitative parts required to build a self-study report (customizable formats to suit multiple accreditation agencies including ABET).
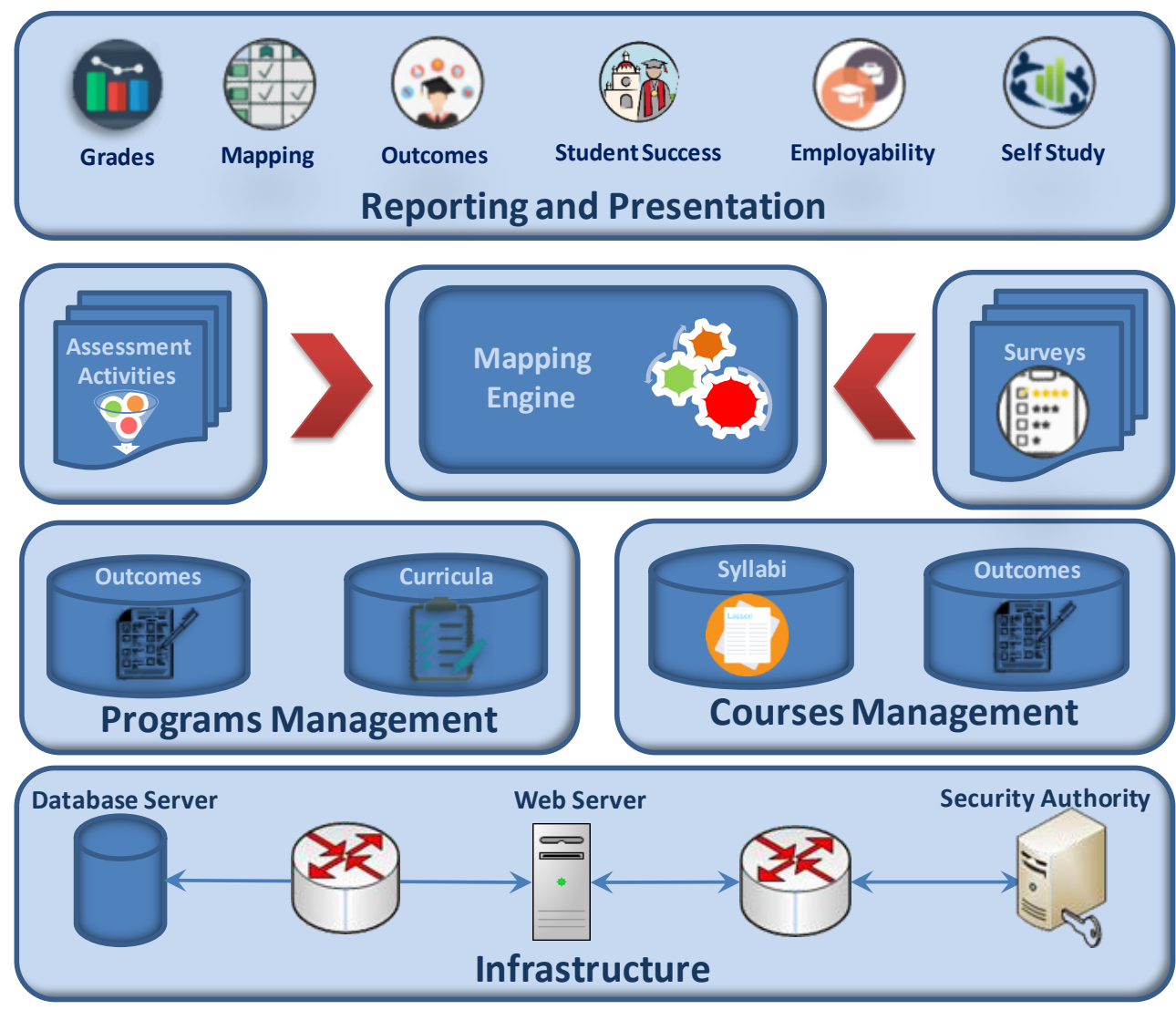

Figure 1. Architectural overview of the web-based ecosystem 


\subsection{Course Grading Tool}

The course grading tool is at the apex of the student assessment process. It is designed to map activities and elements of the adopted student assessment to the course outcomes (COs) set in the syllabi tool. At the beginning of a semester, the course coordinator creates all assessment activities and elements within this tool and maps the elements (questions, projects, components, etc.) to relevant course outcomes which lead to determining how each student and the entire class perform against the respective intended COs. By the end of the course, the overall section assessment report is generated. The instructor uses this report to analyze the results and propose corrective actions. The course grading tool provides templates for instructors to record student and course related data. At the beginning of the semester, the course coordinator creates the course grading system and grade distribution to be used by all course sections instructors.

The process of preparation of an activity within the grading tool is shown in Figure 2. It details the activity elements and their weighted mapping to the course COs. This mapping will be used to assess the student's performance against the intended COs and the course against the overall program student outcomes (PSOs). The process starts with the selection of the desired course from the list of active courses and adding a default grading system. Every activity can be edited to map its elements to COs.

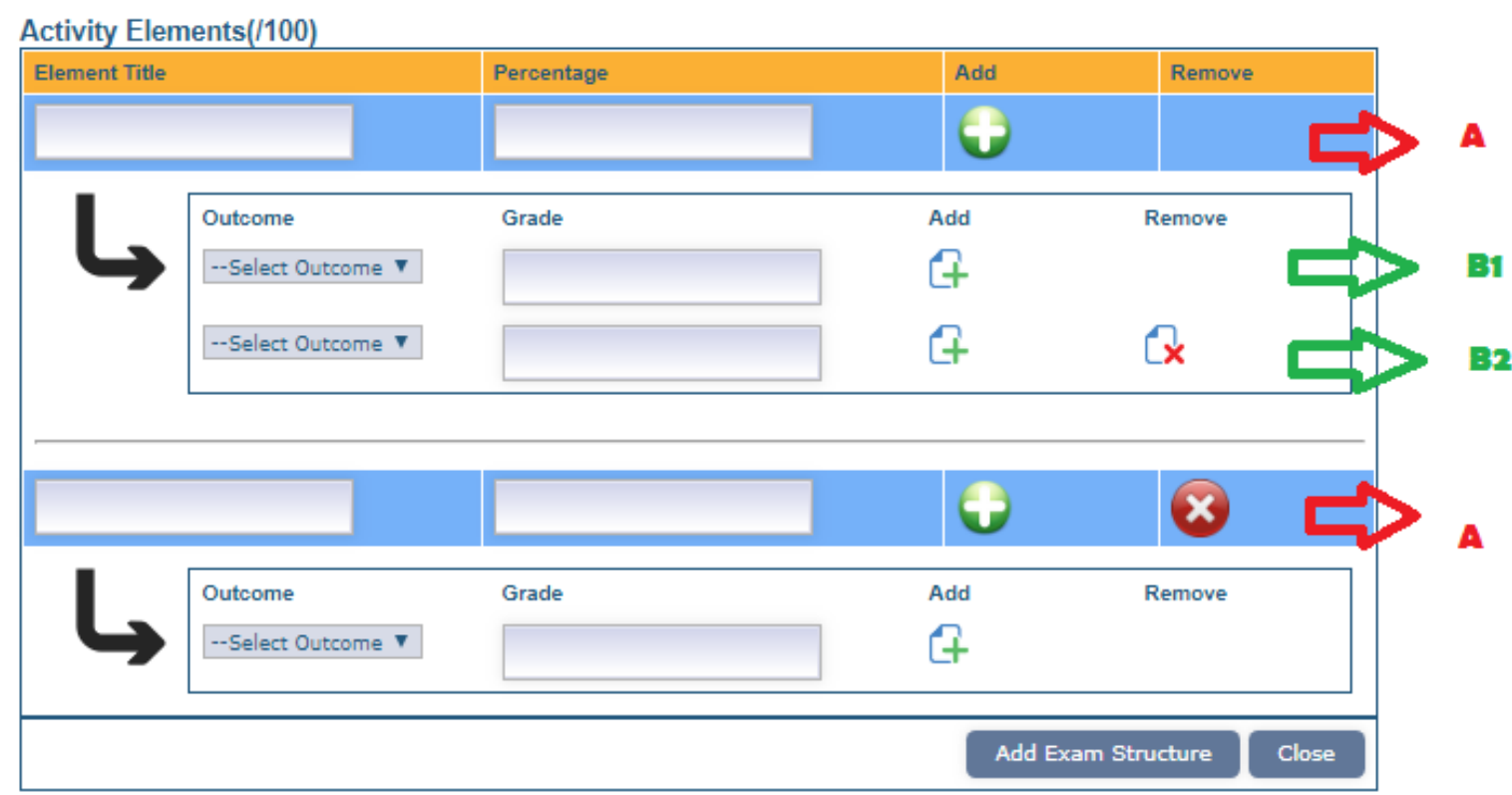

Figure 2. Mapping elements of activities to COs

Exam elements can be added as needed (A), but the summation of all elements must be equal to 100 . For each activity element, associated outcomes can be added (B1 and B2) as needed and the summation of outcomes grades must be equal to element percentage. Once mapping is set, the exam structure can be applied to all offered sections of the course.

\subsection{Coordinator Statistical Tool}

The statistical grading tool is designed to collect metadata from the course grading tool of all sections offered within one campus or across all campuses in a multi-campus university given that the course activities are common and their coordination is centralized. It provides statistics related to a specific course and allows the coordinator to view the completed activities and the elements of each activity. It also offers the coordinator the possibility to select either an activity or an element of an activity and view the grades of the selected activity/element for all the offered course sections. For a course activity, the coordinator selects the campus(es), the school in which the course is offered, the course, and the desired activity. For an element of course activity, the coordinator selects the campus(es), the school in which the course is offered, the course, 
the activity, and the desired element as illustrated in Figure 3. This provides the course coordinator with statistics on a specific activity (See Figure 4) or element within a specific activity for individual course sections and all offered course sections (See Figure 5).

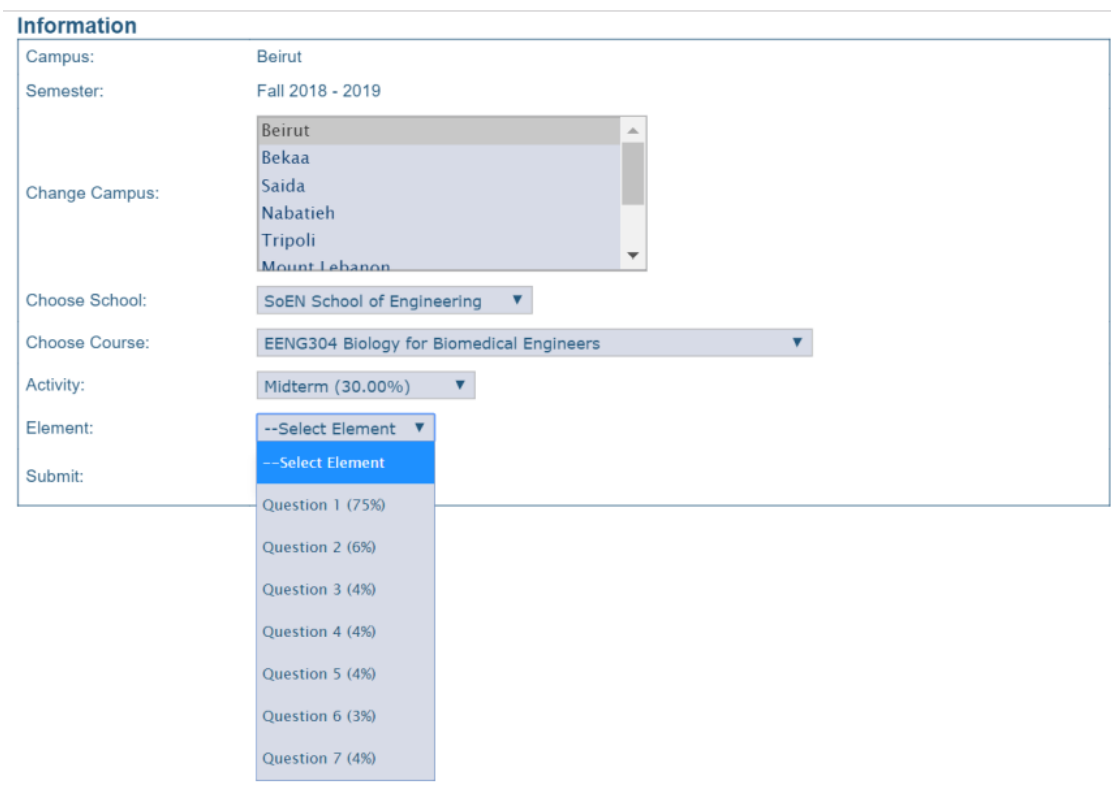

Figure 3. Coordinator statistical tool interface for statistics of elements

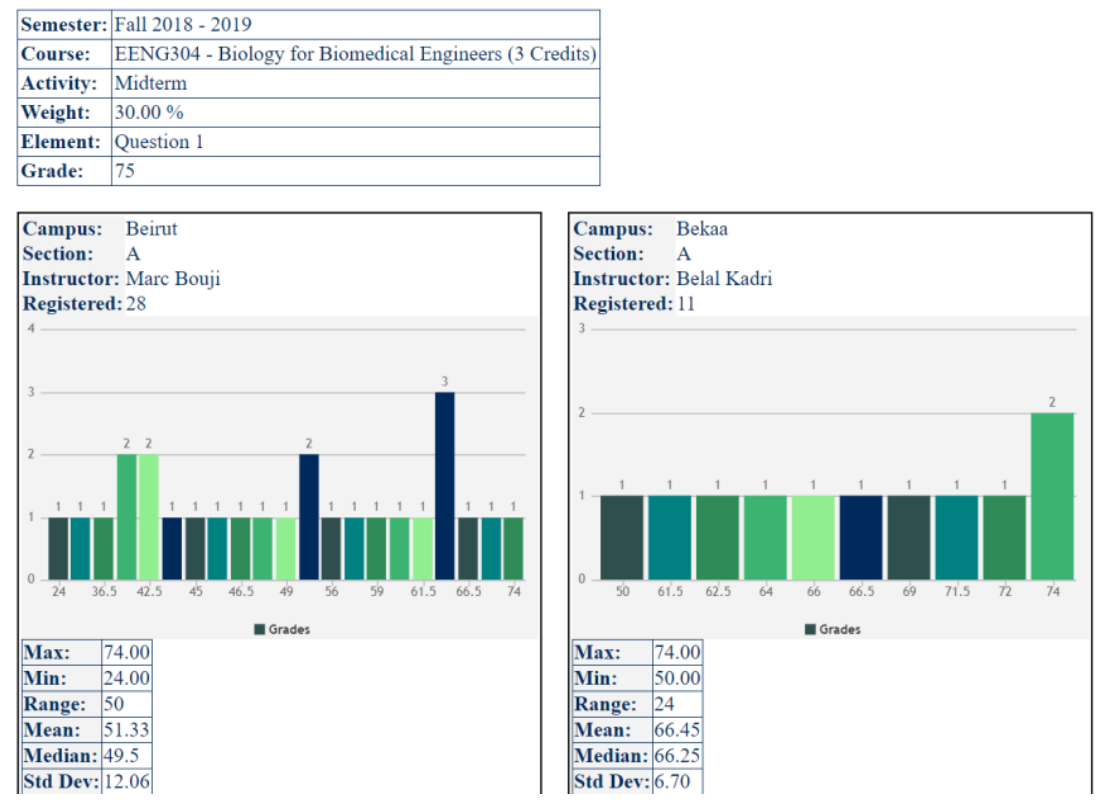

Figure 4. Statistics of a specific activity for individual course sections 


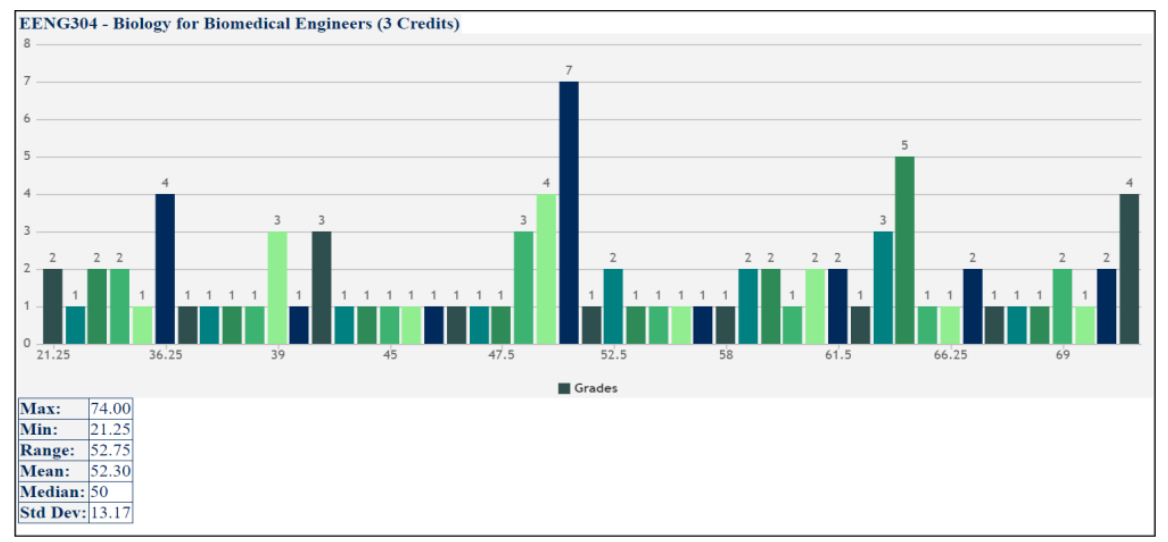

Figure 5. Statistics of an element for all offered sections of a specific course

\subsection{Statistical Analysis}

In order to illustrate the functionality of the presented tools and for a detailed study of the student performance in final examinations, a statistical analysis was conducted on thirty one sections of the Electric Circuits course (EENG250) across nine campuses. The sections are distributed as follows: 11 sections in Beirut campus, 2 sections in Akkar campus, 3 sections in Bekaa campus, 3 sections in Nabatieh campus, 2 sections in Tyre campus, 3 sections in Rayak campus, 2 sections in Saida campus, 2 sections in Tripoli campus, 1 section in Mount Lebanon. Box-plot was selected because it is an efficient tool to compare grades between sections of each campus and between campuses.

Figure 6 (a) shows the comparative box-plots of the eleven sections in Beirut campus that display the final grades for the different sections. For example, $25 \%$ of grades are lower than 78 and about $75 \%$ is above 78 ( since the first quartile Q1 is equal to 78) for section $\mathrm{A}$. In section $\mathrm{K}, 25 \%$ of grades are lower than 50 , $50 \%$ are between 50 and 70 and about $75 \%$ are between 50 and 95 (since the first quartile Q1 and third quartile Q3 are respectively equal to 50 and 70 for section $\mathrm{K}$ ). By comparing these two box plots (Section $\mathrm{A}$ and section $\mathrm{K}$ ), the coordinator can examine the difference grades in these two sections.

Additionally, we can get an idea about the central tendency of each box by observing the position of the median. If the medians are in the center, we can judge the symmetric distribution (normal distribution) and the grades will be evenly split at the median (sections B, E, H and K). If most of the grades are concentrated on the low end of the scale, the distribution (skew-right normal) is skewed right (sections A, C, I and J); and If they are concentrated on the high end of the scale, the distribution (skew-left normal) is skewed left (sections D, G and F).

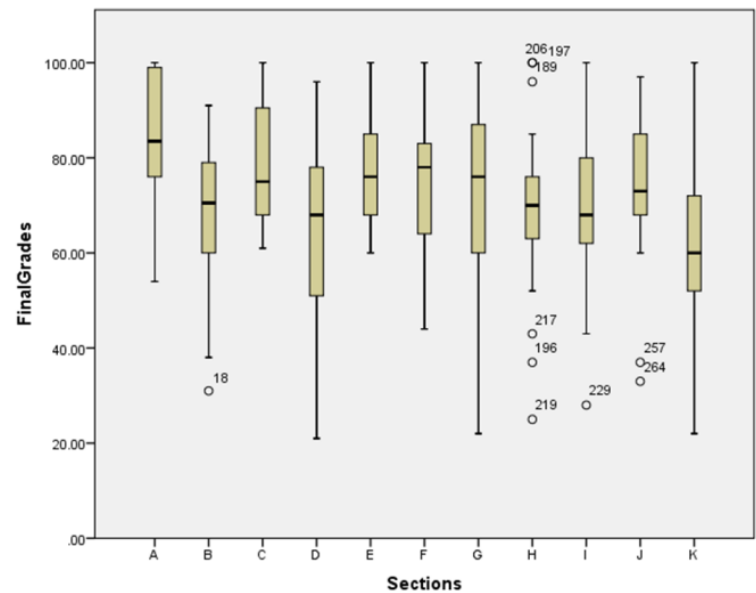

(a)

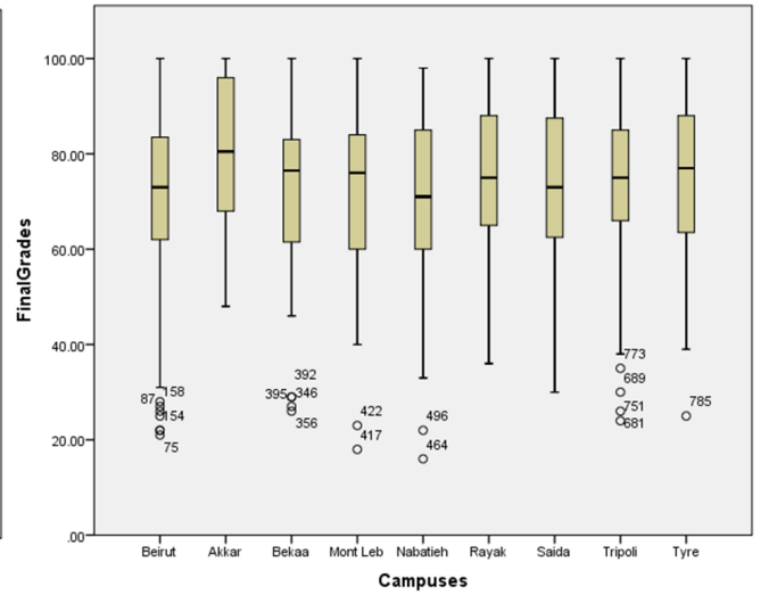

(b)

Figure 6. Box-plots for eleven sections in Beirut campus 
Consequently, this plot shows that there is a variety of different box-plot shapes and positions. Section A appears to have a higher median and section $\mathrm{K}$ a lower median than the other sections. Using the length of the box and the length of the "whiskers", it is possible to estimate the variability of grades for each section and give respectively an idea of the size of the tail of the distribution. Section $\mathrm{H}$ is centered on 68 and having box length smaller than the other boxes and this indicates less variability of grades comparing to the other sections whereas sections $\mathrm{G}$ and $\mathrm{D}$ are the most heterogeneous sections since the length of boxes are greater than the others. So, a wider box indicates more variability and a narrower box indicates little variability in grades. Box plots are used also to detect the outliers in the data, each point outside the whiskers is an outlier. Sections B, H, I and J contain outliers which are bigger or smaller than the rest of the grades. Some of the outliers are extreme $(189,197,206,219,257$ and 257) and other not extreme $(18,196,217,229)$.

Box-plots can also be drawn to compare the final grades between campuses. Figure 6 (b) shows the box plots of grades for all campuses. All campuses have almost the same medians and distributions (normal distribution) but campus Akkar has a median value little higher than the other campuses. We can conclude directly from this figure that final grades in all campuses have the same center value and they are normally distributed in the same way. Furthermore, the medians of all section fall within the yellow-box values for the other box plots, so the difference between sections is not significant.

Box-plots are a simple but powerful graphing tool that provides a lot of information in a simple graph. They can be added to coordinator statistics sheets to facilitate the analysis of grades and to use for reporting results clearly and briefly. They provide a quick way for examining the variation present in the grades, detect any outliers and make decisions based on evidence. They can be used in place of histograms (especially for the sections). Indeed, histograms require a sample size of at least 30 to be useful whereas box-plots require a sample size of only 5, provide more details in the tails of the distribution and are more easily compared across three or more campuses.

\section{CONCLUSION}

The presented online course section grading and statistical tools have been developed, tested, and deployed successfully. The tools use an outcomes-based approach that helps in aligning course elements and activities with course learning outcomes and consequently with program student outcomes. This will facilitate and prepare the floor for university programs to become ABET accredited. The main benefit of having such tools is to have a process-driven organization. Unfortunately, most organizations are not process oriented; they are focused on tasks, jobs, people and structures, but not on processes (Hussein, Bazzi, Dayekh, and Hassan, 2013). Having a complete ecosystem will provide the needed infrastructure to support the organization become process-oriented.

Our future work will concentrate on developing a tool that provides the coordinator with average scores recorded by each one of the COs and PSOs for all sections across all campuses. This enables the coordinator to assess the effect of the previous corrective actions and make decisions based on the statistical analysis of the generated reports. This dynamic and adaptive process lays the foundation of closing the loop of continuous improvement of student learning.

\section{REFERENCES}

Accreditation Board for Engineering and Technology (ABET) Web Site, www.abet.org.

Clark, M.C., Froyd, J., Merton, P. and Richardson, J., 2004. The evolution of curricular change models within the foundation coalition. Journal of Engineering Education, Vol. 93, No. 1, pp. 37-47.

Hajj-Hassan, M., Hussein, B., Chahine, K. and Haj-Ali, A., 2014. A sustainable approach for program students outcomes assessment: the case of the Department of Biomedical Engineering at the Lebanese international university. Journal of Modern Education Review, Vol. 7, No. 4, pp. 555-63.

Hazen, B.T., Wu, Y. and Sankar, C.S., 2012. Factors that influence dissemination in engineering education. IEEE Transactions on Education, Vol. 55, No. 3, pp. 384-393.

Hussein B., Abdul-Nabi S., Merhi Z., Haj-Ali A., 2015. A Web-Based University Courses Syllabi Generator, Journal of Computer Engineering and Intelligent Systems, Vol. 6, No.11, pp. 15-21. 